\title{
Diphoton production in association with two bottom jets
}

\author{
Daniel Fäh, Nicolas Greiner ${ }^{\mathrm{a}}$ \\ Physik Institut, Universität Zürich, Winterthurerstr. 190, 8057 Zürich, Switzerland
}

Received: 31 July 2017 / Accepted: 10 October 2017 / Published online: 7 November 2017

(C) The Author(s) 2017. This article is an open access publication

\begin{abstract}
We study the production of a photon pair in association with two bottom jets at the LHC. This process constitutes an important background to double Higgs production with the subsequent decay of the two Higgs bosons into a pair of photons and $b$-quarks respectively. We calculate this process at next-to-leading order accuracy in QCD and find that QCD corrections lead to a substantial increase of the production cross section due to new channels opening up at nextto-leading order and their inclusion is therefore inevitable for a reliable prediction. Furthermore, the approximation of massless $b$-quarks is scrutinized by calculating the process with both massless and massive $b$-quarks. We find that the massive bottom quark leads to a substantial reduction of the cross section where the biggest effect is, however, due to the use of a four-flavor PDF set and the corresponding smaller values for the strong coupling constant.
\end{abstract}

\section{Contents}

1 Introduction . . . . . . . . . . . . . . 1

2 Calculational setup ............ . . 2

3 Numerical results . . . . . . . . . . . . . 2

3.1 Cuts and parameter settings . . . . . . . . 2

3.2 Cross sections and differential distributions . . 2

3.3 Massive $b$-quarks . . . . . . . . . . . 6

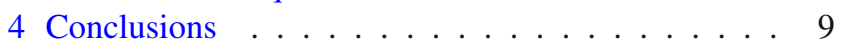

References ............... 9

\section{Introduction}

The boson discovered at the LHC $[1,2]$ seems to be in very good agreement with prescription of a Standard Model like Higgs boson. In the Standard Model the Higgs mass is the only free parameter in the theory and its precise determina-

a e-mail: greiner@physik.uzh.ch tion was one of the main experimental targets [3]. Furthermore, the Standard Model predicts the shape of the Higgs potential, so a measurement of the parameters of the potential will allow us to discriminate a Standard Model Higgs boson from various BSM scenarios. This however requires the measurement of the Higgs self coupling, which can be measured in Higgs boson pair production processes. The value of the Higgs mass allows for measurements in a variety of decay channels and both ATLAS and CMS have performed studies to measure the Higgs self coupling, e.g. in the decay channels $\gamma \gamma b \bar{b}[4-7], b \bar{b} b \bar{b}[6,8-11], \gamma \gamma W W^{*}, b \bar{b} W W^{*}, \tau^{+}{ }^{-} b \bar{b}$ [6, 12-18].

From a Standard Model calculational point of view, the signal process (i.e. the production of a Higgs boson pair) is known at leading order in the full theory [19-21], and in various approximations taking higher order corrections into account [22-31]. Only very recently the NLO result taking the full top mass dependence into account became available $[33,34]$.

In this paper we focus on one possible decay channels, namely where one Higgs decays into a pair of photons, whereas the second decays into a pair of $b$-quarks. This process can be seen as a compromise between a four $b$-quark signal and a four photon signature. The first would benefit from a large $H \rightarrow b \bar{b}$ branching ratio but suffers from a large irreducible QCD background, whereas the latter exhibits a very clean signal with four photons, but suffers from a very small $H \rightarrow \gamma \gamma$ branching ratio.

In the case of massless $b$-quarks the process $\gamma \gamma b \bar{b}$ can be seen as a subset of the process $\gamma \gamma j j$, which is known at NLO in QCD [35-37]. The main motivation for the general two jet process was, however, more to assess the background of a single Higgs in VBF production rather than focusing on final state $b$-quarks. As we will see, the tagging of two final state $b$-jets significantly alters the behavior of the higher order corrections and therefore this process cannot be directly compared to the general two jet process. 
The paper is organized as follows. In Sect. 2 we describe the setup that has been used to obtain the numerical results which we discuss in Sect. 3. Finally we conclude in Sect. 4.

\section{Calculational setup}

The NLO corrections are calculated by combining the two automated programs GoSAM $[38,39]$ for the generation and evaluation of the virtual one-loop amplitudes, and the Monte Carlo event generator SHERPA [43]. The combination between the two is realized using the standardized Binoth Les Houches Accord [44,45].

GoSAM is based on an algebraic approach where $d$ dimensional integrands are generated using Feynman diagrams. It uses QGRAF [40] and FORM [41,42] for the diagram generation, and SPINNEY [46] and FORM to write an optimized Fortran output. For the reduction of the tensor integrals we used NINJA [47-49], which carries out the reduction on the integrand level in a fully automated way via Laurent expansion. Alternatively one can choose other reduction strategies such as the OPP reduction method [50-52], which is implemented in SAMURAI [53] or methods based on tensor integral reduction as implemented in GoLEM95 [54-57]. For the evaluation of the remaining scalar integrals we have used ONELOOP [58].

The evaluation of all tree-level like matrix elements within SHERPA has been performed using COMIX [59], the subtraction terms have been calculated with the SHERPA's implementation of the Catani-Seymour dipole formalism $[60,61]$.

\section{Numerical results}

In the following we present numerical results for the LHC with a center of mass energy of $\sqrt{s}=13 \mathrm{TeV}$. To assess $b$-mass effects the calculation has been carried out with both massless $b$-quarks in the five-flavor scheme as well as with massive $b$-quarks in the four-flavor scheme.

\subsection{Cuts and parameter settings}

For the massless case we have used the CT10nlo pdf set [62] and the CT10nlo_nf4 set for the massive case respectively. In the massive case the $b$-mass has been set to $4.7 \mathrm{GeV}$.

Renormalization and factorization scales are set to be equal and the central scale was chosen to be

$\mu_{R}=\mu_{F}=\frac{1}{2} \sqrt{m_{\gamma \gamma}^{2}+\left(\sum_{i} p_{T, i}\right)^{2}}$,

where the sum runs over the final state partons. As this process contains external photons the electroweak coupling con- stant $\alpha$ is set to $\alpha=1 / 137.03599976$. We have included top-quark loops in the virtual corrections with a top mass of $m_{t}=171.2 \mathrm{GeV}$.

The presence of final state photons requires the application of a photon isolation criterion to render the NLO corrections finite. We employed a smooth cone isolation criterion [63] with the following parameters:

$R=0.4, \quad \epsilon=0.05, \quad n=1$.

For tight isolation the smooth cone isolation has been shown to be in good agreement with the alternative approach of applying a photon fragmentation function (see e.g. [68]). Additionally the isolated photons are required to fulfill

$p_{T, \gamma}>30 \mathrm{GeV}, \quad\left|\eta_{\gamma}\right|<2.5$.

The QCD partons are clustered with an anti- $k_{T}$ algorithm [64] contained in the Fastjet package [65]. The jet radius has been set to $R=0.4$ and events where both $b$-quarks are clustered into a jet are rejected in order to ensure that there are at least two $b$-jets present in the final state. For the jets we require

$p_{T, j}>20 \mathrm{GeV}, \quad\left|y_{j}\right|<4.4$.

\subsection{Cross sections and differential distributions}

We start the discussion of the numerical results with the case of a massless $b$-quark. We assess the theoretical uncertainty by usual scale variation of a factor of 2 around the central scale. Based on the cuts and settings described above we find for the total cross section

$\sigma_{L O}=38.6_{-17 \%}^{+22 \%} \mathrm{fb}, \quad \sigma_{\mathrm{NLO}}=56.2_{-15 \%}^{+20 \%}$.

From Eq. (5) one can see that the NLO corrections enhance the total cross section by almost 50 per cent. It also shows that the theoretical uncertainty does not improve at NLO, instead for both LO and NLO one obtains an uncertainty of 15-20\% in each direction when varying the scale by a factor of 2 . This situation is shown more explicitly in Fig. 1 where we show the cross section as a function of the scale for a broader range. Looking at the curve for the inclusive NLO result one sees a born-like behavior even at NLO. In particular no reduction on the scale dependence is obtained throughout the whole range of scales. The typical turnover that one expects at NLO is not present.

A special feature of this process is that the leading order process is mediated by two types of initial state, the $q \bar{q}$ and the $g g$-channel. At NLO, however, also the quark-gluon channel is opening up in the real emission. In order to investigate whether it is this channel that is responsible for the 


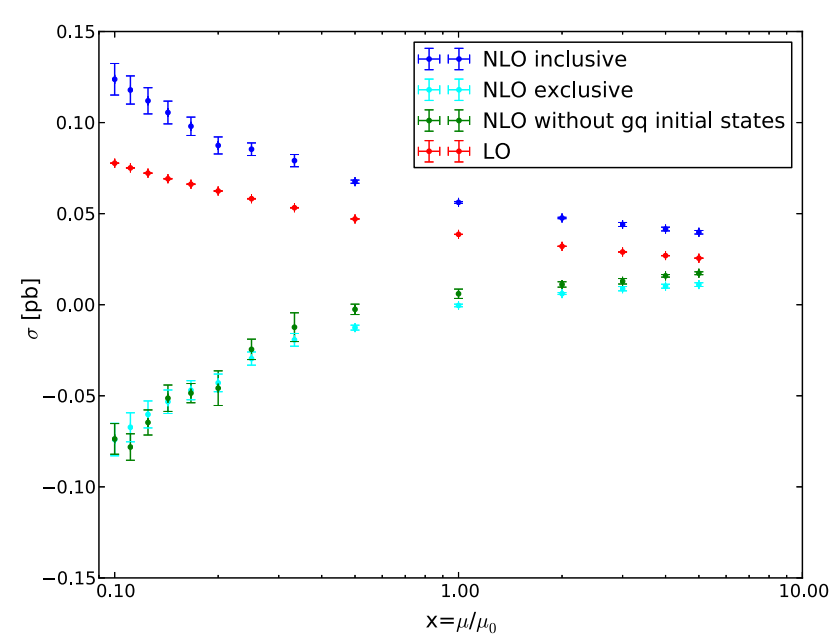

Fig. 1 Total cross sections at LO and NLO for massless $b$-quarks. In addition it shows the NLO cross section where the initial $q g$-channel has been removed as well as the exclusive two $b$-jet cross section where a veto on a third jet has been imposed

tree-level like behavior we made two different checks. First, we completely remove the quark-gluon channel from the process (green curve). This has a tremendous impact on the NLO result rendering the corrections negative over the whole range and the absolute value increases when going to smaller scales which even leads to unphysical negative cross sections for scales smaller than the central scale. Removing a production channel is of course not a physical meaningful procedure but it shows that this channel is indeed responsible for the behavior of the inclusive NLO cross section. A physically well-defined strategy, however, is to impose a jet veto on a possible third jet. This means that we remove events where a third QCD parton (independent whether it is a $b$-quark or not) would form an independent jet applying the anti-kt algorithm and which passes the jet cuts in Eq. (4). A jet veto effectively cuts away an intrinsically positive contribution from the real emission and will therefore lead to a decrease of the NLO result. The exclusive NLO result is given by the turquoise curve. Interestingly the two approaches lead to very similar results. Even though vetoing a jet is a well-defined procedure it leads to negative cross sections for scales smaller than the central scale. This behavior is driven by the virtual corrections that yield a negative correction and their absolute value increases when going to smaller scales. For small scales they dominate the total cross section and supersede the leading order contribution. This general behavior is expected and it indicates that the central scale could be chosen to be larger although it has been proven to be a good choice for the general diphoton plus two jets process [35]. The general two jet process of [35] is, however, different in that respect that the $q g$-channel already occurs at leading order which explains the different behavior of the total cross section under scale variation.
A lowering of the veto threshold would lead to a further decrease of the total cross section as one cuts away a larger part of the positive real emission contribution. It is clear that imposing a jet veto raises the question to what extent possible resummation effects can change the result and the associated theoretical uncertainty. This is, however, beyond the scope of this paper. The results show that this process is highly sensitive to an additional jet veto and that the scale variation might therefore not be an accurate measure of the theoretical uncertainty. The inclusive NLO result seems, however, suitable as a conservative estimation.

We now turn to a discussion of the differential distributions. Fig. 2 shows the $p_{T}$ distribution of the two leading $b$-jets, where the jets are $p_{T}$-ordered. For both jets the NLO corrections for low values of $p_{T}$ are relatively small which means that the NLO result agrees with the leading order result within the systemic uncertainty. Also the size of the NLO uncertainty is reduced compared to the leading order uncertainty. Going higher in $p_{T}$, however, very rapidly increases the NLO corrections and from the order of $100 \mathrm{GeV}$ on the differential $k$-factors are in the range of 2-2.5. Also the size of the NLO uncertainty band increases and for values beyond $\sim 100 \mathrm{GeV}$ the uncertainties at NLO are roughly twice as big as the LO ones.

For the transverse momentum distribution of the photons shown in Fig. 3 the behavior is less pronounced than for the jets. For the leading photon the corrections are smallest for low values of $p_{T}$ and rise almost linearly with increasing transverse momentum leading to a $k$-factor of almost 2 for values around $500 \mathrm{GeV}$. Similar to the jet distributions there is basically no overlap between the uncertainty bands. The subleading photon shows a milder behavior compared to the leading photon. Although the uncertainty bands also hardly overlap the differential $k$-factor is flat to a good approximation. For both photons one sees that the size of the NLO uncertainty is roughly of the same size as the LO uncertainties whereas for the jets the NLO uncertainties were larger except for small values of $p_{T}$.

As this process constitutes a background to double Higgs production, the invariant mass distributions are also essential. In the upper row of Fig. 4 we show the invariant masses of the two leading $b$ - jets and of the two photons. In both cases one observes a significant shape distortion by the NLO corrections. They exhibit large corrections at low values followed by a minimum in the range of $60-80 \mathrm{GeV}$. In the case of the jets the NLO corrections then increase roughly linearly again, leading to substantial corrections for invariant masses beyond say $200 \mathrm{GeV}$. For the photons this behavior is mitigated and the differential $k$-factor is flat to a good approximation in the mass range beyond $200 \mathrm{GeV}$. It is worth noting that in the range around the Higgs mass the corrections are rather mild and one still finds an overlap between the uncertainty bands. And in particular the NLO behavior 

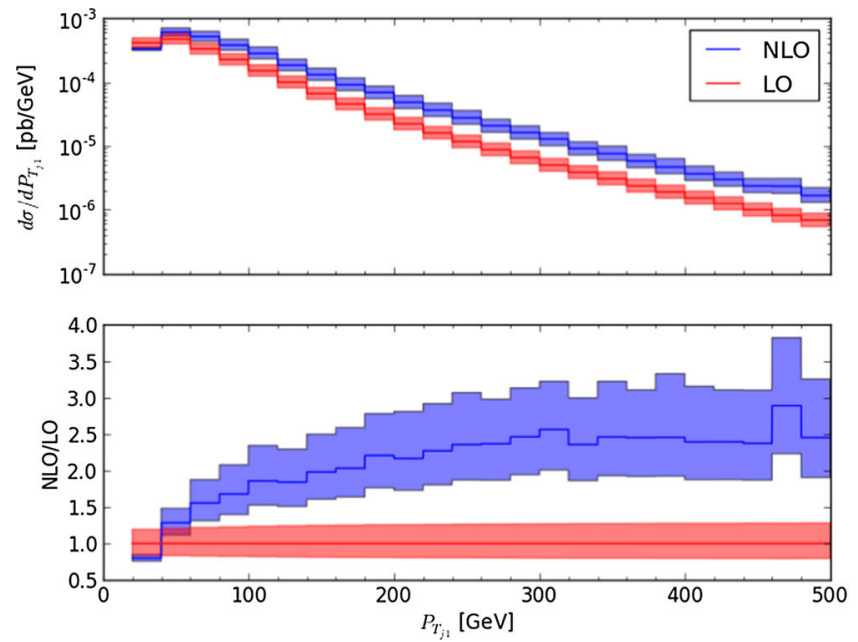

Fig. 2 Transverse momentum of the two leading $b$-jets
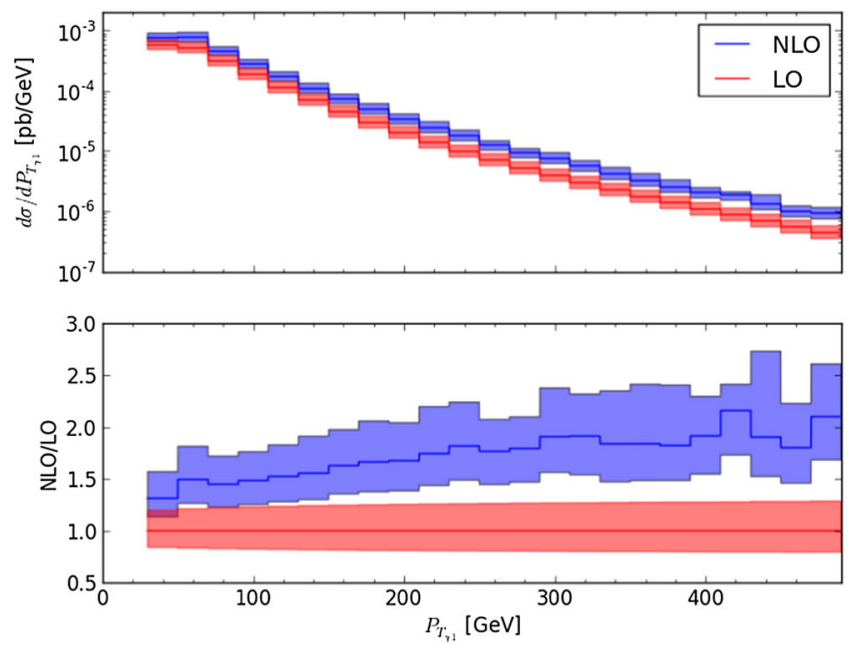

Fig. 3 Transverse momentum of the two photons

for low invariant masses allows one to reduce the NLO corrections by imposing an appropriate cut around the Higgs mass. The increase in the low invariant mass region at NLO can be understood by keeping in mind that additional radiation decreases the available phase space for each particle and the two photons or two jets, respectively, become closer in terms of both energy and angular separation. In a region where the invariant masses are small they are more sensitive to extra radiation and therefore one observes a shift towards small invariant masses. The plot in the lower row of Fig. 4 shows the total invariant mass of the final state where the sum runs over the two photons and the jets. There we see a drastic change in the shape of the distribution when going from leading order to next-to-leading order. At low invariant masses the NLO corrections are negative and substantial but then increase linearly and lead to substantial positive corrections in the region above $\sim 400 \mathrm{GeV}$. With the additional quark-
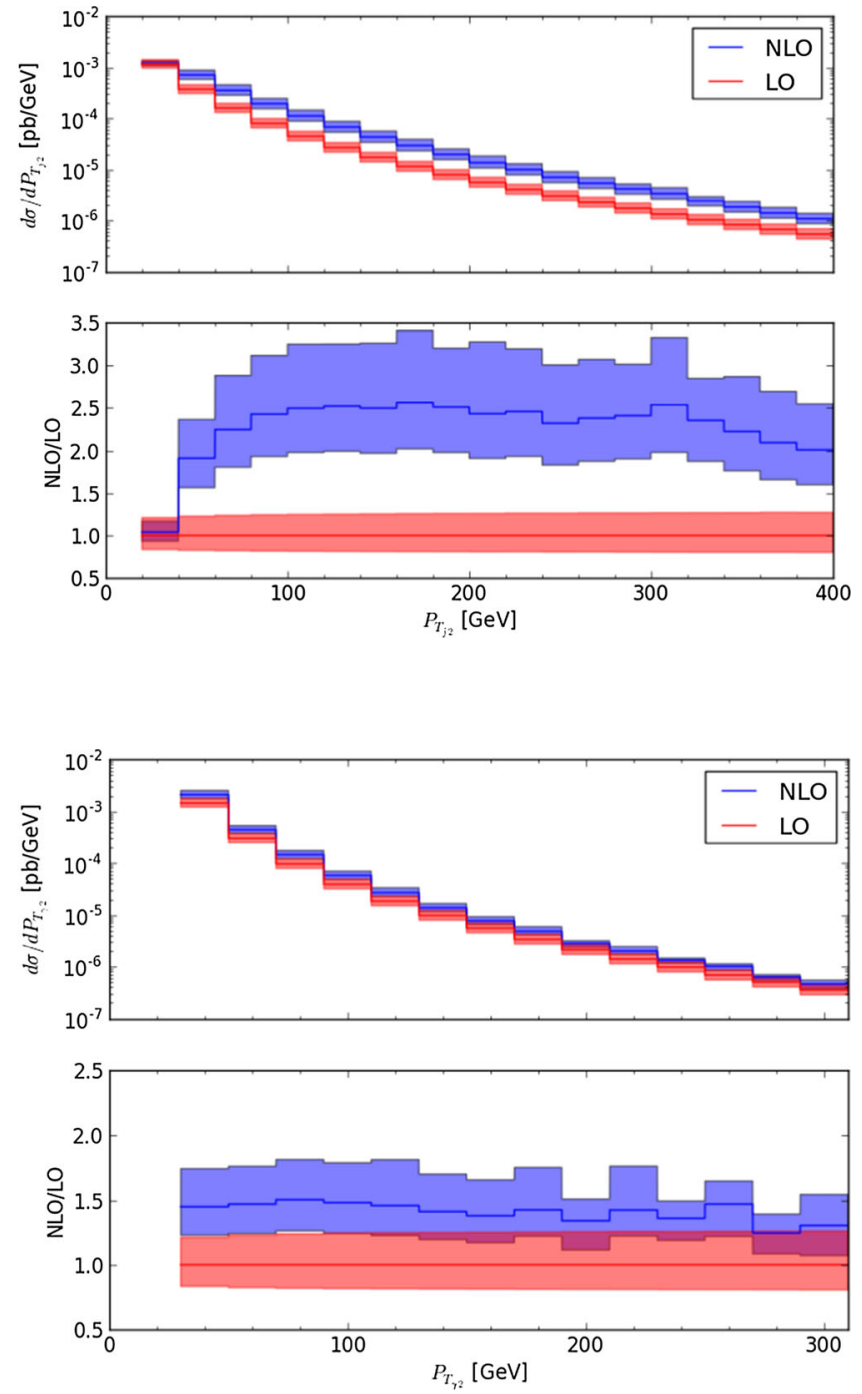

gluon channel in the real radiation it is not surprising that the kinematics of the process changes compared to the leading order behavior, and this observable, being very inclusive in the final state probes the underlying kinematics of the process. In addition, for high center of mass energies (and this is essentially what this observable shows) it is more likely to have a third jet than for small center of mass energies. The real emission contribution will therefore play a more important role for high invariant masses whereas for small invariant masses the chance that the event is rejected increases as the available energy is distributed among more particles which increases the chance that one or more particles might not pass the cuts. One can expect also differences between signal and background in various angular distributions as in the case of the signal the $b$-jets and the photons stem from the decay of a spin- 0 particle, whereas for the background processes the angular correlations are different. Figure 5 shows the $R$ - 

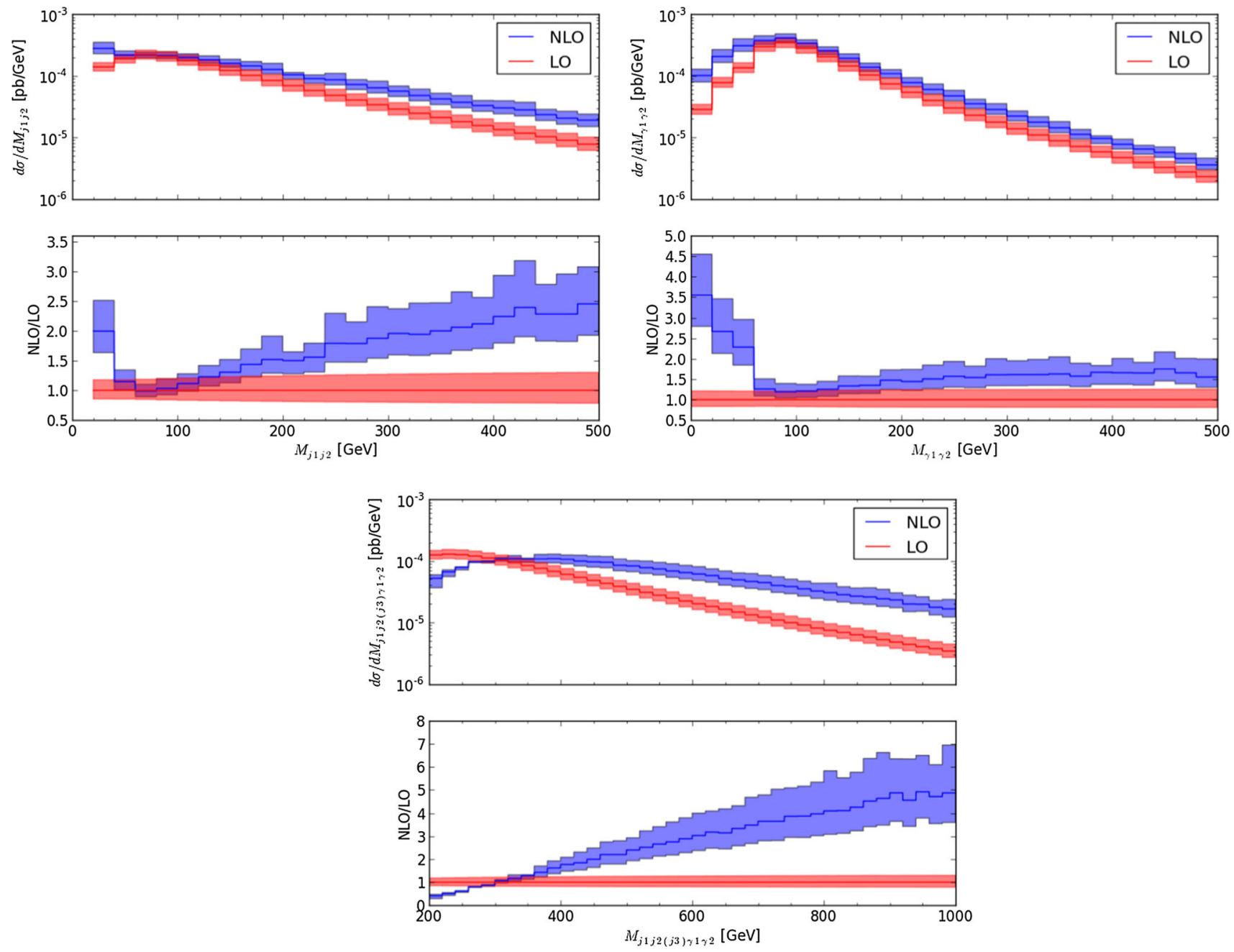

Fig. 4 Invariant mass distributions of the two leading jets (upper left), the two photons (upper right) and the total invariant mass
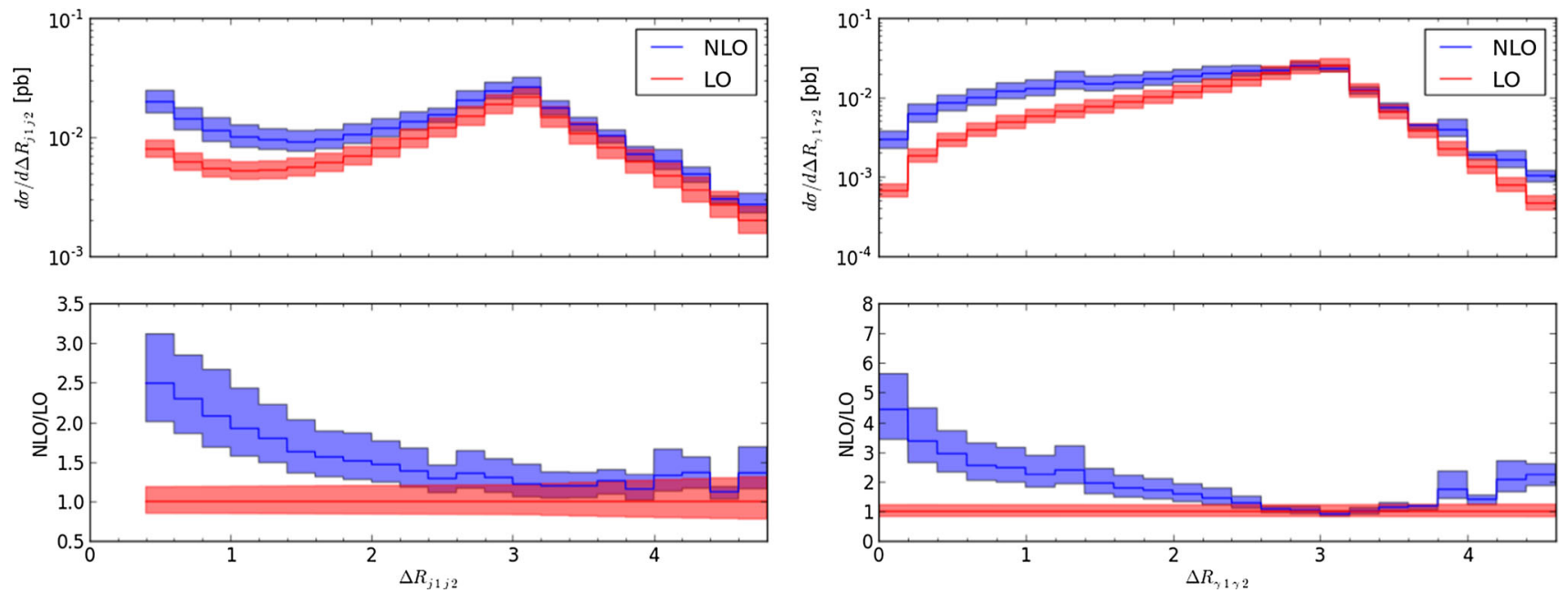

Fig. $5 R$-separation of the two leading jets (1.h.s.) and the two photons (r.h.s.) 

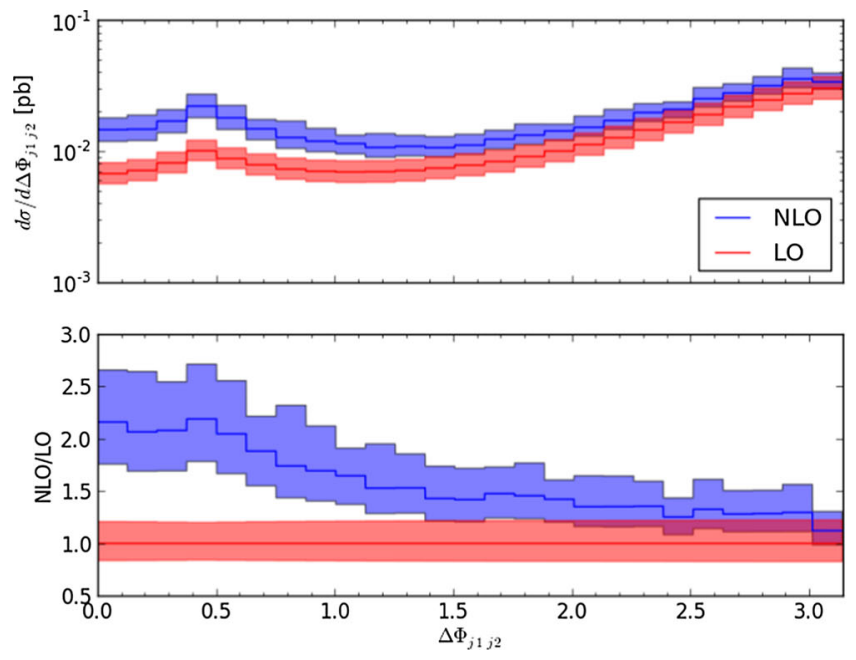
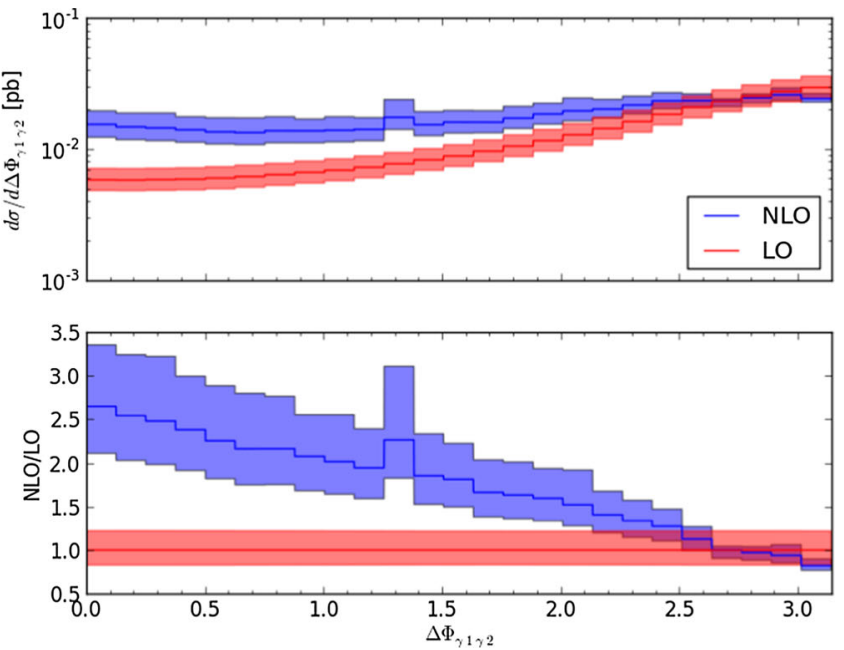

Fig. 6 Azimuthal separation of the two leading jets (1.h.s.) and the two photons (r.h.s.)

separation between the two leading jets and the two photons respectively. Both distributions exhibit large corrections for small values for the separation with a minimum around $\pi$. A similar behavior is also found for the azimuthal angle which is shown in Fig. 6 for the leading jets (1.h.s.) and the two photons (r.h.s.). Also there one finds for both the jets and the photons the largest contributions for small angles followed by a constant decrease. For $\Delta \phi \approx \pi$ the NLO result agrees with the LO result within the theoretical uncertainty. For Figs. 5 and 6 concerning the increase for small values at NLO the same argument as for the invariant masses of the two photons and the two leading $b$ jets can be applied. Additional radiation leads to a decrease of angular separation among all particles which leads to an enhancement of small separation at NLO.

\subsection{Massive $b$-quarks}

In this section we will scrutinize the validity of treating the $b$-quark as a massless particle. For this purpose we set the mass of the $b$-quark to its pole mass of $4.7 \mathrm{GeV}$. For a consistent treatment we employ the four-flavor scheme and use the CT10nlo_nf4 pdf set. Table 1 shows the total cross sections for the central scale at LO and NLO for massive quarks in direct comparison to the massless results. The massive LO order result is reduced by $\sim 10 \%$, at NLO the massive result is $\sim 16 \%$ smaller than the massless result. This reduces the $k$-factor by $\sim 7 \%$.

At first it may seem unreasonable that the introduction of the $b$-mass does have such an influence on the result given that the mass is relatively small compared to all other scales in this process. However, one should keep in mind that there are several effects that need to be taken into account. The biggest effect certainly comes from the change of the pdf
Table 1 Total cross sections at LO and NLO for the central scale and for massless and massive $b$-quarks

\begin{tabular}{lll}
\hline$\mu=\mu_{0}$ & $m_{b}=0 \mathrm{GeV}$ & $m_{b}=4.7 \mathrm{GeV}$ \\
\hline$\sigma_{\mathrm{LO}}[\mathrm{fb}]$ & $38.62(2)$ & $34.83(1)$ \\
$\sigma_{\mathrm{NLO}}[\mathrm{fb}]$ & $56.2(4)$ & $47.4(4)$ \\
$k=\frac{\sigma_{N L O}}{\sigma_{L O}}$ & 1.46 & 1.36 \\
\hline
\end{tabular}

set that comes along with a lower value of $\alpha_{s} \cdot \alpha_{s}\left(M_{Z}\right)$ is $\sim 4.5 \%$ smaller in the massive case. This effect is the driving force in the reduction of the cross section. In addition, for this process the subprocesses with initial state $b$-quarks are enhanced due to t-channel like diagrams with the $b$-quark line going from initial to final state. These type of diagrams yield a large contribution that enhances the importance of initial state $b$-quarks compared to the other sea-quark contributions. A sample of diagrams that are only present in the five-flavor scheme but not in the four-flavor scheme is shown in Fig. 7.

This effect has also been observed in the context of multiple $b$-quark production $[66,67]$ and also there the overall effect has been found to be large [69]. From comparing LO order results within the four-flavor scheme for the massless and the massive case we estimate the pure mass effect contributing to $\sim 40 \%$ of the reduction of the cross section.

It is now also important to investigate if and how big the massive $b$-quark will affect differential distributions. For simplicity we present the massive results only for the central scale. The focus here is on the change of the shape caused by the mass effects and we assume that the theoretical uncertainty will be of a very similar size as for the massless case. In Fig. 8 we show the transverse momentum distribution for the two leading jets. The upper ratio plot shows the ratio of the massive LO contribution over the massless result, the lower ratio plot shows the same for the NLO result. For compari- 

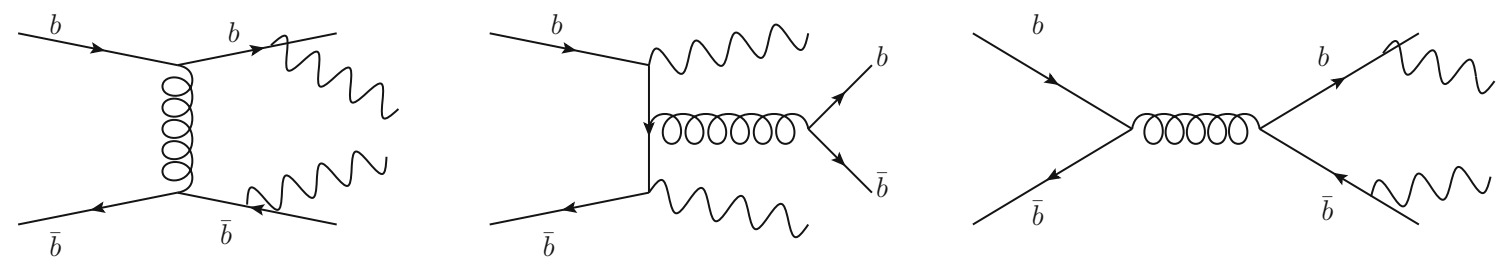

Fig. 7 Sample of tree-level diagrams that are only present in the five-flavor scheme and not in the four-flavor scheme
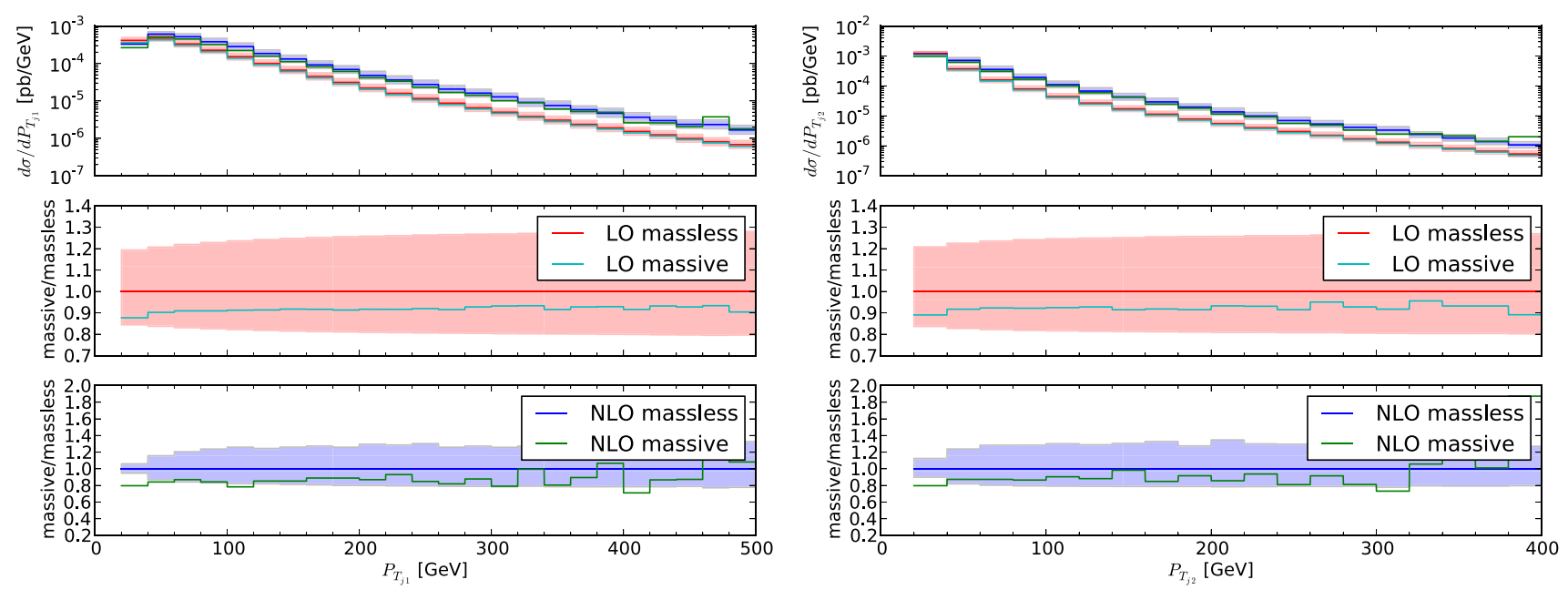

Fig. 8 Transverse momentum distribution of the two leading jets for massive $b$-quarks
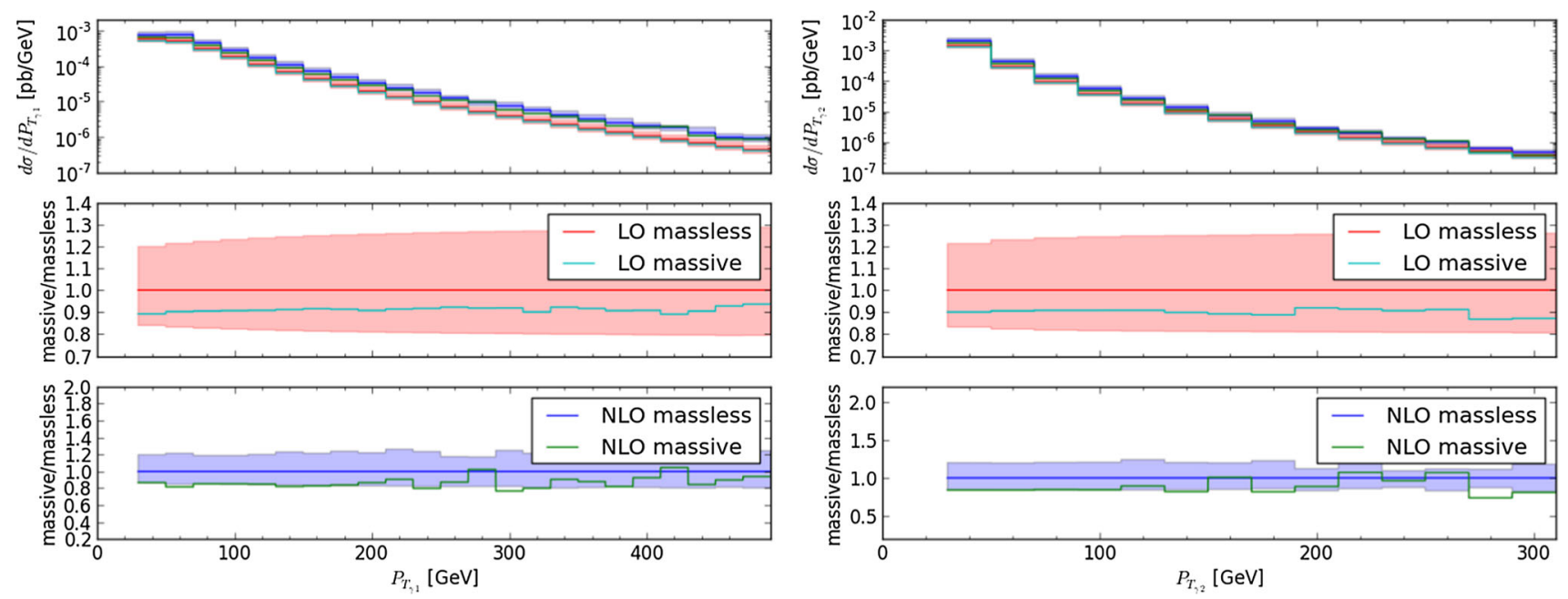

Fig. 9 Transverse momentum distribution of the two photons for massive $b$-quarks

son we also show the scale uncertainty for the massless case. The mass effects are dominated by the general decrease of the cross section in the massive case, but the differential $k$-factor is flat to a quite good approximation and the central scale of the massive result is still within the uncertainty band of the massless result except for the first bin where the uncertainty band becomes smaller. One can therefore conclude that the uncertainty from setting the mass to a non-zero value is contained within the systematic uncertainty from scale variation.
The transverse momentum distribution of the two photons shows exactly the same behavior as can be seen in Fig. 9. Also here the differential $k$-factor is flat to a good approximation and the massive result is still in agreement within the uncertainty of the massless result. The same is also true for the invariant masses of the two leading jets and the two photons as can be seen in Fig. 10. Also here the massive result can be incorporated in the systematic uncertainty of the massless calculation. For the total invariant mass shown in the lower 

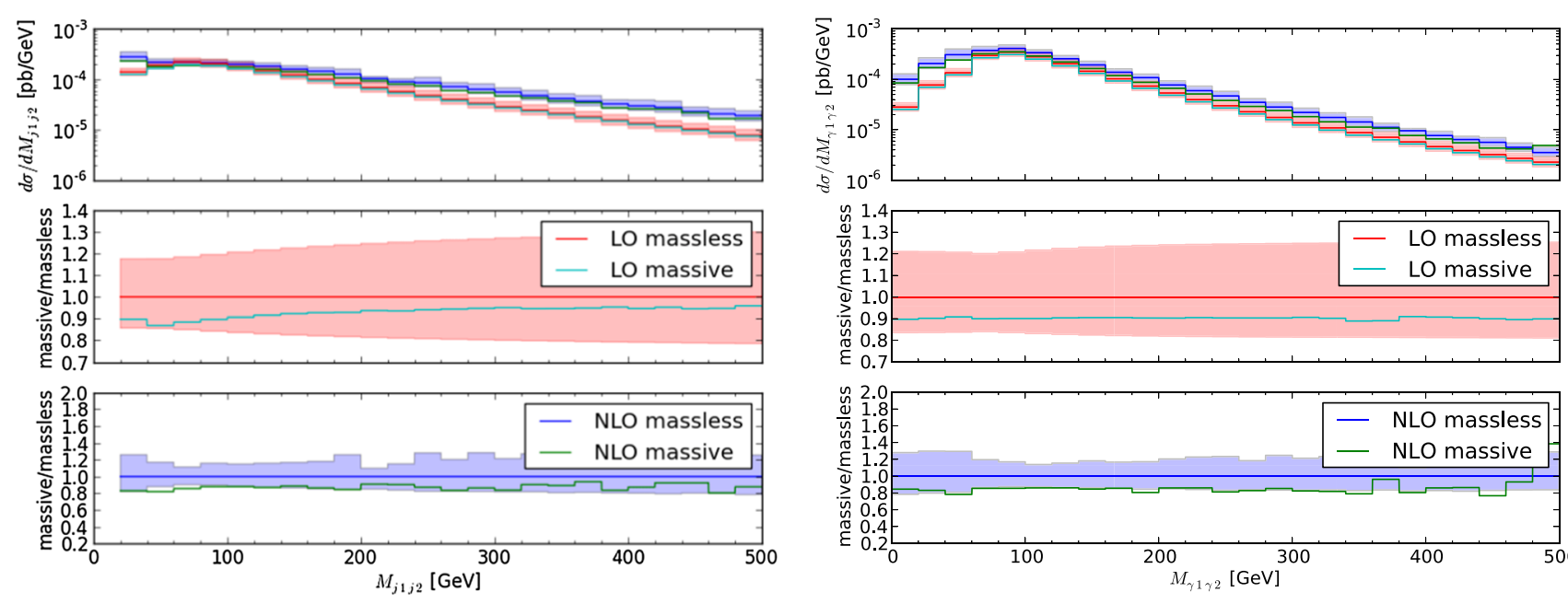

\& 10
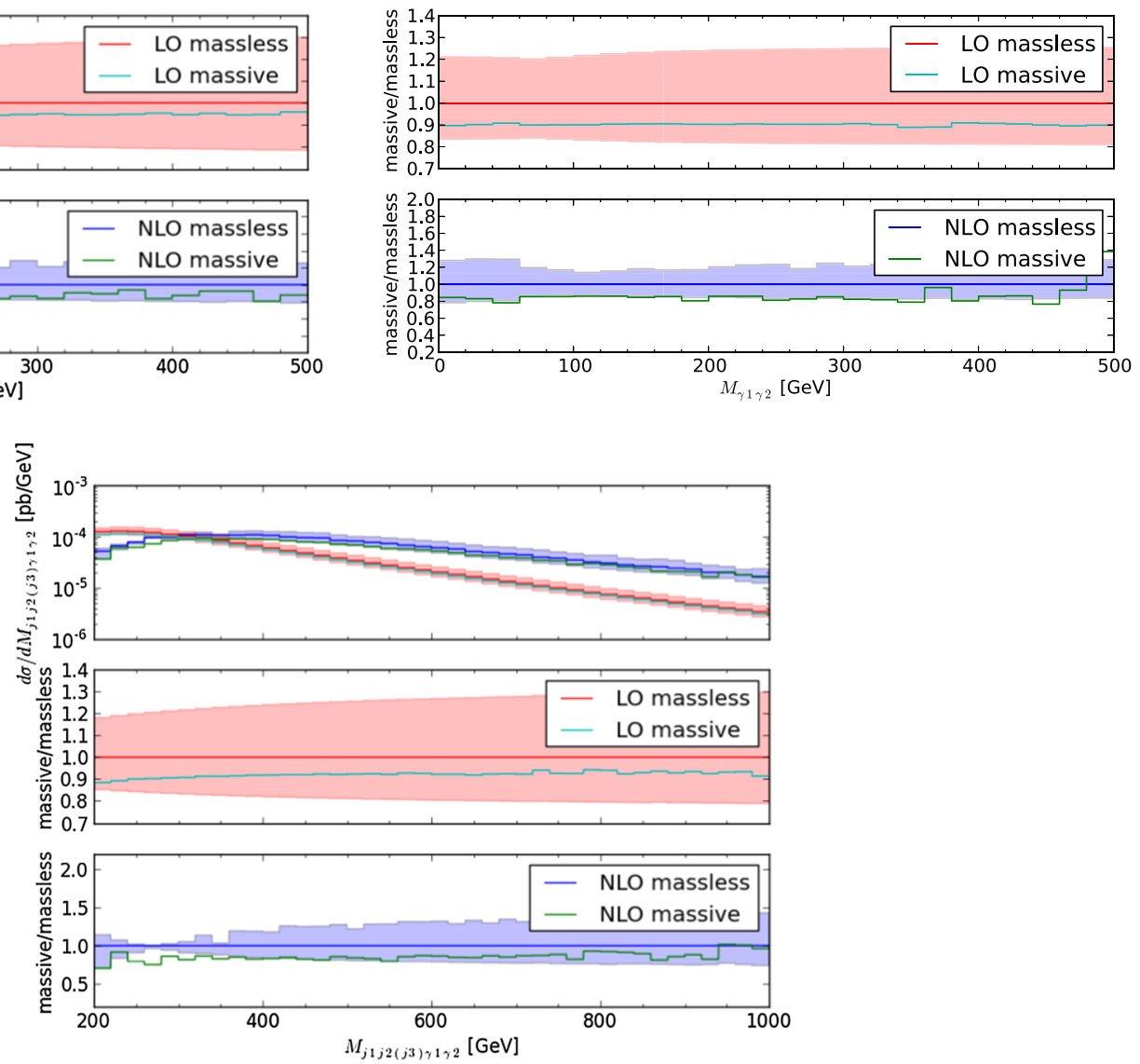

Fig. 10 Invariant mass distribution for the two leading jets and the two photons (upper row) and for the total invariant mass (lower row) for massive $b$-quarks

row of Fig. 10 the situation is a bit more special. Also here the mass leads to a flat shift downwards, but the ratio plots shows that for the NLO result the error band becomes very small in the region between 250 and $300 \mathrm{GeV}$. The reason for this behavior is that the upper and the lower scale cross the central scale in that region which makes the scale uncertainty vanish and leaving the massive result outside the estimated uncertainty. This might also be interpreted such that for this observable our scale choice is not suitable to describe this particular observable and give a reliable estimation of the underlying uncertainties. In general on would expect that if the introduction of a massive $b$-quark yields a shape distortion compared to the massless case, then this should preferably show up in distributions that separate regions of low and high energy/transverse momentum, such that there are regions where the $b$-mass becomes large compared to the other scales in the process. Distributions like the transverse momenta of the $b$-jets or the invariant mass of the dijet sys- tem seem to be the ideal candidates. However, as we have seen above, even in these distributions we do not observe a significant shape distortion and the effects of the $b$-mass are essentially reduced to a global shift induced by the different value of $\alpha_{s}$. It is therefore not surprising that also in angular distributions we do not observe a different pattern. We exemplify this by showing the angular separation between the two leading jets and the two photons in Fig. 11. As for the transverse momentum distribution of the jets one observes a small effect in the first bin where the massive result is slightly below the uncertainty band of the massless result but also here the differential $k$-factor is flat over the whole range. For the separation between the photons the situation is very similar to the massive result being at the lower end of the uncertainty band with an otherwise flat $k$-factor.

In summary, the inclusion of the $b$-mass has a substantial effect on the total cross section and on differential distributions. However, it leads just to a global shift towards 

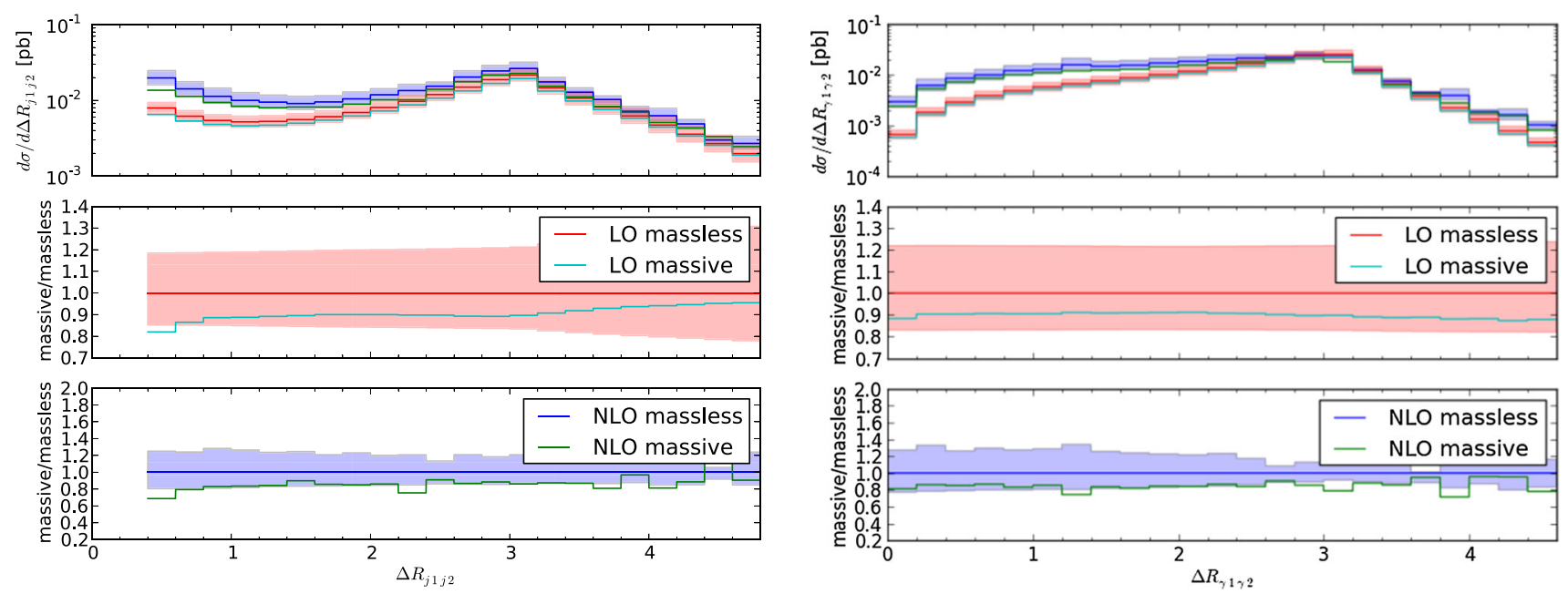

Fig. 11 Angular separation between the two leading jets (1.h.s.) and the two photons (r.h.s.) for massive $b$-quarks

smaller values largely caused by the four-flavor pdf set and the smaller value for $\alpha_{s}$. But it does not lead to significant distortions of shapes of the differential distributions. A shift via a global $k$-factor would therefore be able to accurately describe the mass effect.

\section{Conclusions}

The measurement of the triple Higgs coupling is an essential ingredient to completely determine the structure of the Higgs potential and to answer the question whether the Higgs boson is in agreement with the prediction from the Standard Model. The production of two Higgs bosons via gluon fusion yields the biggest contribution that includes the triple Higgs vertex.

In this paper we investigated the background of one of the most import decay channels, where one Higgs would decay into a $b \bar{b}$ pair and the other Higgs would decay into a pair of photons. We calculated the $\mathcal{O}\left(\alpha_{s}^{2} \alpha^{2}\right)$ contribution at next-toleading order in QCD in the fully automated Sherpa + GoSam setup. We found large corrections due to new partonic channels opening up for the real emission contribution leading to a tree-level like behavior of the cross section under variation of renormalization- and factorization scale. The inclusion of NLO effects is therefore viable for a reliable theoretical prediction.

We also assessed the impact of a massive bottom quark. In a consistent treatment the inclusion of the mass comes along with a four-flavor scheme pdf set and therefore also the removal of subprocesses with initial state $b$-quarks. Altogether we found a significant reduction of the cross section which, however, is largely caused by the pdf set and the smaller value of $\alpha_{s}$. The actual mass only plays a minor role. The massive result is still contained within the systematic uncertainty of the massless one and the shapes of the differ- ential distributions are unchanged to a good approximation. This means that the mass effects can effectively be described by applying a global $k$-factor to the massless results.

Acknowledgements We would like to thank Marek Schoenherr for his help with Sherpa and Thomas Gehrmann and Gudrun Heinrich for various useful discussions. NG was supported by the Swiss National Science Foundation under contract PZ00P2_154829.

Open Access This article is distributed under the terms of the Creative Commons Attribution 4.0 International License (http://creativecomm ons.org/licenses/by/4.0/), which permits unrestricted use, distribution, and reproduction in any medium, provided you give appropriate credit to the original author(s) and the source, provide a link to the Creative Commons license, and indicate if changes were made.

Funded by SCOAP ${ }^{3}$.

\section{References}

1. ATLAS Collaboration, G. Aad et al., Observation of a new particle in the search for the Standard Model Higgs boson with the ATLAS detector at the LHC. Phys. Lett. B716, 1-29 (2012). arXiv: 1207.7214

2. CMS Collaboration, S. Chatrchyan et al., Observation of a new boson at a mass of $125 \mathrm{GeV}$ with the CMS experiment at the LHC. Phys.Lett. B716, 30-61 (2012). arXiv:1207.7235

3. ATLAS, CMS Collaboration, G. Aad et al., Combined Measurement of the Higgs Boson Mass in $p p$ Collisions at $\sqrt{s}=7$ and 8 TeV with the ATLAS and CMS Experiments. Phys. Rev. Lett. 114, 191803 (2015). arXiv: 1503.07589

4. ATLAS Collaboration, Search for Higgs boson pair production in the $b \bar{b} \gamma \gamma$ final state using pp collision data at $\sqrt{s}=13 \mathrm{TeV}$ with the ATLAS detector. ATLAS-CONF-2016-004, CERN (Geneva, 2016)

5. CMS Collaboration, V. Khachatryan et al., Search for two Higgs bosons in final states containing two photons and two bottom quarks. arXiv:1603.06896

6. ATLAS Collaboration, G. Aad et al., Searches for Higgs boson pair production in the $h h \rightarrow b b \tau \tau, \gamma \gamma W W^{*}, \gamma \gamma b b, b b b b$ channels with the ATLAS detector. Phys. Rev. D92, 092004 (2015). arXiv: 1509.04670 
7. ATLAS Collaboration, G. Aad et al., Search For Higgs Boson Pair Production in the $\gamma \gamma b \bar{b}$ Final State using $p p$ Collision Data at $\sqrt{s}=8 \mathrm{TeV}$ from the ATLAS Detector. Phys. Rev. Lett. 114(8), 081802 (2015). arXiv: 1406.5053

8. ATLAS Collaboration, M. Aaboud et al., Search for pair production of Higgs bosons in the $b \bar{b} b \bar{b}$ final state using proton-proton collisions at $\sqrt{s}=13 \mathrm{TeV}$ with the ATLAS detector. arXiv: 1606.04782

9. CMS Collaboration, Search for resonant pair production of Higgs bosons decaying to two bottom quark-antiquark pairs in protonproton collisions at $13 \mathrm{TeV}$, CMS-PAS-HIG-16-002, (2016)

10. CMS Collaboration, V. Khachatryan et al., Search for resonant pair production of Higgs bosons decaying to two bottom quark antiquark pairs in proton-proton collisions at $8 \mathrm{TeV}$. Phys. Lett. B749, 560-582 (2015). arXiv: 1503.04114

11. ATLAS Collaboration, G. Aad et al., Search for Higgs boson pair production in the $b \bar{b} b \bar{b}$ final state from pp collisions at $\sqrt{s}=8$ TeVwith the ATLAS detector. Eur. Phys. J. C75(9), 412 (2015). arXiv: 1506.00285

12. ATLAS Collaboration, Search for Higgs boson pair production in the final state of $\gamma \gamma W W^{*}(\rightarrow l v j j)$ using $13.3 \mathrm{fb}^{-1}$ of $p p$ collision data recorded at $\sqrt{s}=13 \mathrm{TeV}$ with the ATLAS detector. ATLASCONF-2016-071 (2016)

13. CMS Collaboration, Search for Higgs boson pair production in the $\mathrm{b} \bar{b} \mathrm{l} v \mathrm{l} v$ final state at $\sqrt{s}=13 \mathrm{TeV}$. CMS-PAS-HIG-16-024 (2016)

14. CMS Collaboration, Search for non-resonant Higgs boson pair production in the bbtautau final state using 2016 data. CMS-PAS-HIG16-028 (2016)

15. CMS Collaboration, Search for resonant Higgs boson pair produc-

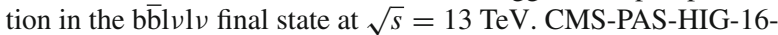
011 (2016)

16. C.M.S. Collaboration, Search for resonant Higgs boson pair production in the $b \bar{b} \tau^{+} \tau^{-}$final state, CMS-PAS-HIG-16-013 CERN (Geneva, 2016)

17. CMS Collaboration, C. Collaboration, Search for non-resonant Higgs boson pair production in the $b \bar{b} \tau^{+} \tau^{-}$final state, CMS-PASHIG-16-012 (2016)

18. CMS Collaboration, Model independent search for Higgs boson pair production in the $\mathrm{b} \overline{\mathrm{b}} \tau^{+} \tau^{-}$final state. Tech. Rep. CMS-PASHIG-15-013 (2016)

19. O.J.P. Eboli, G.C. Marques, S.F. Novaes, A.A. Natale, Twin Higgs boson production. Phys. Lett. B 197, 269 (1987)

20. E.W.N. Glover, J.J. van der Bij, Higgs Boson pair production via Gluon Fusion. Nucl. Phys. B 309, 282 (1988)

21. T. Plehn, M. Spira, P.M. Zerwas, Pair production of neutral Higgs particles in gluon-gluon collisions. Nucl. Phys. B479, 46-64 (1996). arXiv: hep-ph/9603205. Erratum: Nucl. Phys.B531,655(1998)

22. S. Dawson, S. Dittmaier, M. Spira, Neutral Higgs boson pair production at hadron colliders: QCD corrections. Phys. Rev D58, 115012 (1998). arXiv:hep-ph/9805244

23. F. Maltoni, E. Vryonidou, M. Zaro, Top-quark mass effects in double and triple Higgs production in gluon-gluon fusion at NLO. JHEP 11, 079 (2014). arXiv: 1408.6542

24. J. Grigo, J. Hoff, K. Melnikov, M. Steinhauser, On the Higgs boson pair production at the LHC. Nucl. Phys B875, 1-17 (2013). arXiv: 1305.7340

25. J. Grigo, K. Melnikov, M. Steinhauser, Virtual corrections to Higgs boson pair production in the large top quark mass limit. Nucl. Phys. B888, 17-29 (2014). arXiv:1408.2422

26. J. Grigo, J. Hoff, M. Steinhauser, Higgs boson pair production: top quark mass effects at NLO and NNLO. Nucl. Phys B900, 412 (2015). arXiv: 1508.00909

27. G. Degrassi, P.P. Giardino, R. Grber, On the two-loop virtual QCD corrections to Higgs boson pair production in the Standard Model. Eur. Phys. J C76(7), 411 (2016). arXiv:1603.00385
28. D. de Florian, J. Mazzitelli, Two-loop virtual corrections to Higgs pair production. Phys. Lett. B724, 306-309 (2013). arXiv: 1305.5206

29. D. de Florian, J. Mazzitelli, Higgs Boson pair production at next-tonext-to-leading order in QCD. Phys. Rev. Lett. 111, 201801 (2013). arXiv: 1309.6594

30. D.Y. Shao, C.S. Li, H.T. Li, J. Wang, Threshold resummation effects in Higgs boson pair production at the LHC. JHEP 07, 169 (2013). arXiv:1301.1245

31. D. de Florian, Higgs pair production at next-to-next-to-leading logarithmic accuracy at the LHC. JHEP 09, 053 (2015). arXiv: 1505.07122

32. D. de Florian, M. Grazzini, C. Hanga, S. Kallweit, J. M. Lindert, P. Maierhöfer, J. Mazzitelli, D. Rathlev, Differential Higgs Boson pair production at next-to-next-to-leading order in QCD. arXiv: 1606.09519

33. S. Borowka, N. Greiner, G. Heinrich, S. Jones, M. Kerner, J. Schlenk, U. Schubert, T. Zirke, Higgs Boson Pair Production in Gluon Fusion at Next-to-Leading Order with Full Top-Quark Mass Dependence, Phys. Rev. Lett. 117(1), 012001 (2016). Erratum ibid 079901. arXiv:1604.06447

34. S. Borowka, N. Greiner, G. Heinrich, S.P. Jones, M. Kerner, J. Schlenk, T. Zirke, JHEP 1610, 107 (2016). https://doi.org/10.1007/ JHEP10(2016)107. arXiv:1608.04798 [hep-ph]

35. T. Gehrmann, N. Greiner, G. Heinrich, Phys. Rev. Lett. 111, 222002 (2013). https://doi.org/10.1103/PhysRevLett.111.222002. arXiv:1308.3660 [hep-ph]

36. S. Badger, A. Guffanti, V. Yundin, JHEP 1403, 122 (2014). https:// doi.org/10.1007/JHEP03(2014)122. arXiv:1312.5927 [hep-ph]

37. Z. Bern, L.J. Dixon, F. Febres Cordero, S. Hoeche, H. Ita, D.A. Kosower, N.A. Lo Presti, D. Maitre, Phys. Rev. D 90(5), 054004 (2014). https://doi.org/10.1103/PhysRevD.90. 054004. arXiv:1402.4127 [hep-ph]

38. G. Cullen, N. Greiner, G. Heinrich, G. Luisoni, P. Mastrolia et al., Automated one-loop calculations with GoSam. Eur.Phys.J C72, 1889 (2012). arXiv: 1111.2034

39. G. Cullen et al., GOSAM-2.0: a tool for automated one-loop calculations within the Standard Model and beyond. Eur. Phys. J. C74(8), 3001 (2014). arXiv: 1404.7096

40. P. Nogueira, Automatic Feynman graph generation. J. Comput. Phys. 105, 279-289 (1993)

41. J. Vermaseren, New features of FORM. arXiv: math-ph/0010025

42. J. Kuipers, T. Ueda, J. Vermaseren, J. Vollinga, FORM version 4.0. Comput. Phys. Commun 184, 1453-1467 (2013). arXiv: 1203.6543

43. T. Gleisberg, S. Hoeche, F. Krauss, M. Schonherr, S. Schumann, F. Siegert, J. Winter, JHEP 0902, 007 (2009). https://doi.org/10. 1088/1126-6708/2009/02/007. arXiv:0811.4622 [hep-ph]

44. T. Binoth et al., Comput. Phys. Commun 181, 1612 (2010). https:// doi.org/10.1016/j.cpc.2010.05.016. arXiv:1001.1307 [hep-ph]

45. S. Alioli et al., Comput. Phys. Commun 185, 560 (2014). https:// doi.org/10.1016/j.cpc.2013.10.020. arXiv:1308.3462 [hep-ph]

46. G. Cullen, M. Koch-Janusz, T. Reiter, Comput. Phys. Commun 182, 2368 (2011). https://doi.org/10.1016/j.cpc.2011.06.007. arXiv: 1008.0803 [hep-ph]

47. P. Mastrolia, E. Mirabella, T. Peraro, JHEP 1206, 095 (2012). https://doi.org/10.1007/JHEP11(2012)128, 10.1007/ JHEP06(2012)095. Erratum: [JHEP 1211, 128 (2012)]. arXiv:1203.0291 [hep-ph]

48. H. van Deurzen, G. Luisoni, P. Mastrolia, E. Mirabella, G. Ossola, T. Peraro, JHEP 1403, 115 (2014). https://doi.org/10.1007/ JHEP03(2014)115. arXiv:1312.6678 [hep-ph]

49. T. Peraro, Comput. Phys. Commun 185, 2771 (2014). https://doi. org/10.1016/j.cpc.2014.06.017. arXiv:1403.1229 [hep-ph]

50. G. Ossola, C.G. Papadopoulos, R. Pittau, Nucl. Phys. B 763, 147 (2007). https://doi.org/10.1016/j.nuclphysb.2006.11.012. arXiv:hep-ph/0609007 
51. P. Mastrolia, G. Ossola, C.G. Papadopoulos, R. Pittau, JHEP 0806, 030 (2008). https://doi.org/10.1088/1126-6708/2008/06/ 030. arXiv:0803.3964 [hep-ph]

52. G. Ossola, C.G. Papadopoulos, R. Pittau, JHEP 0805, 004 (2008). https://doi.org/10.1088/1126-6708/2008/05/004. arXiv:0802.1876 [hep-ph]

53. P. Mastrolia, G. Ossola, T. Reiter, F. Tramontano, JHEP 1008, 080 (2010). https://doi.org/10.1007/JHEP08(2010)080. arXiv:1006.0710 [hep-ph]

54. G. Heinrich, G. Ossola, T. Reiter, F. Tramontano, JHEP 1010, 105 (2010). https://doi.org/10.1007/JHEP10(2010)105. arXiv:1008.2441 [hep-ph]

55. T. Binoth, J.-P. Guillet, G. Heinrich, E. Pilon, T. Reiter, Comput. Phys. Commun 180, 2317 (2009). https://doi.org/10.1016/j.cpc. 2009.06.024. arXiv:0810.0992 [hep-ph]

56. G. Cullen, J.P. Guillet, G. Heinrich, T. Kleinschmidt, E. Pilon, T. Reiter, M. Rodgers, Comput. Phys. Commun 182, 2276 (2011). https://doi.org/10.1016/j.cpc.2011.05.015. arXiv:1101.5595 [hep$\mathrm{ph}]$

57. J.P. Guillet, G. Heinrich, J.F. von Soden-Fraunhofen, Comput. Phys. Commun 185, 1828 (2014). https://doi.org/10.1016/j.cpc. 2014.03.009. arXiv:1312.3887 [hep-ph]

58. A. van Hameren, Comput. Phys. Commun 182, 2427 (2011). https://doi.org/10.1016/j.cpc.2011.06.011. arXiv:1007.4716 [hep$\mathrm{ph}]$

59. T. Gleisberg, S. Hoeche, JHEP 0812, 039 (2008). https://doi.org/ 10.1088/1126-6708/2008/12/039. arXiv:0808.3674 [hep-ph]

60. S. Catani, M.H. Seymour, Nucl. Phys. B 485, 291 (1997). https://doi.org/10.1016/S0550-3213(96)00589-5, 10.1016/ S0550-3213(98)81022-5. Erratum: [Nucl. Phys. B 510, 503 (1998)]. arXiv: hep-ph/9605323
61. S. Catani, S. Dittmaier, M.H. Seymour, Z. Trocsanyi, Nucl. Phys. B 627, 189 (2002). https://doi.org/10.1016/ S0550-3213(02)00098-6. arXiv:hep-ph/0201036

62. H.L. Lai, M. Guzzi, J. Huston, Z. Li, P.M. Nadolsky, J. Pumplin, C.-P. Yuan, Phys. Rev. D 82, 074024 (2010). https://doi.org/10. 1103/PhysRevD.82.074024. arXiv:1007.2241 [hep-ph]

63. S. Frixione, Phys. Lett. B 429, 369 (1998). https://doi.org/10.1016/ S0370-2693(98)00454-7. hep-ph/9801442

64. M. Cacciari, G.P. Salam, G. Soyez, JHEP 0804, 063 (2008). https:// doi.org/10.1088/1126-6708/2008/04/063. arXiv:0802.1189 [hep$\mathrm{ph}]$

65. M. Cacciari, G.P. Salam, G. Soyez, Eur. Phys. J. C 72, 1896 (2012). https://doi.org/10.1140/epjc/s10052-012-1896-2. arXiv:1111.6097 [hep-ph]

66. T. Binoth, N. Greiner, A. Guffanti, J. Reuter, J.-P. Guillet, T. Reiter, Phys. Lett. B 685, 293 (2010). https://doi.org/10.1016/j.physletb. 2010.02.010. arXiv:0910.4379 [hep-ph]

67. N. Greiner, A. Guffanti, T. Reiter, J. Reuter, Phys. Rev. Lett. 107, 102002 (2011). https://doi.org/10.1103/PhysRevLett.107.102002. arXiv:1105.3624 [hep-ph]

68. T. Gehrmann, N. Greiner, G. Heinrich, JHEP 1306, 058 (2013). https://doi.org/10.1007/JHEP06(2014)076, https://doi. org/10.1007/JHEP06(2013)058. Erratum: JHEP 1406, 076 (2014). arXiv:1303.0824 [hep-ph]

69. G. Bevilacqua, M. Czakon, M. Krämer, M. Kubocz, M. Worek, JHEP 1307, 095 (2013). https://doi.org/10.1007/ JHEP07(2013)095. arXiv:1304.6860 [hep-ph] 